\title{
EVALUACIÓN DEL FACTOR DE CONFIANZA CONSIDERANDO DAÑO ESTRUCTURAL SÍSMICO EN EL TIEMPO
}

\author{
Dante Tolentino López ${ }^{(1)}$ y Sonia E. Ruiz Gómez ${ }^{(2)}$
}

\begin{abstract}
RESUMEN
Se propone un criterio para evaluar el factor de confianza que toma en cuenta la variación tanto de la capacidad estructural como la demanda sísmica, al término de un intervalo de tiempo. El factor de confianza y su correspondiente nivel de confianza se expresan mediante ecuaciones matemáticas cerradas. La formulación se desarrolla de acuerdo con el formato de diseño basado en factores de demanda y capacidad estructural. Las expresiones propuestas se extienden a partir de la formulación original que no considera el deterioro en el tiempo propuesta por Cornell. El criterio propuesto se aplica a un edificio de concreto reforzado de 10 niveles ubicado en suelo blando con características similares al sitio de la Secretaría de Comunicaciones y Transportes en la Ciudad de México.
\end{abstract}

Palabras clave: confiabilidad estructural; factor de confianza; deterioro estructural; daño acumulado

\section{EVALUATION OF THE CONFIDENCE FACTOR CONSIDERING SEISMIC STRUCTURAL DAMAGE OVER TIME}

\begin{abstract}
A criterion to evaluate the confidence factor that takes into account the deterioration of the structural capacity and the increment of the seismic demand at the end of a time interval is proposed. The confidence factor and its confidence level are expressed by means of closed-form mathematical equations. The formulation is made in accordance with the Demand and Capacity Factor Design Format. The simplified closed-form expressions are based on the original formulation proposed by Cornell where the structural deterioration over time is not considered. The proposed criterion is exemplified by means of the analysis of a 10-story building located on soft soil with characteristics similar to those of the site of the Ministry of Communications and Transports in Mexico City.
\end{abstract}

Keywords: structural reliability; confidence factor; structural deterioration; cumulative damage

Artículo ganador del Noveno Concurso Nacional de Tesis de Doctorado 2015 organizado por la SMIS, recibido el 10 de diciembre de 2015 y aprobado para su publicación el 28 de diciembre de 2015. Se aceptarán comentarios y/o discusiones hasta cinco meses después de su publicación.

(1) Profesor Investigador, Sección de Estudios de Posgrado e Investigación, ESIA Zacatenco, Instituto Politécnico Nacional, Gustavo A. Madero, Ciudad de México, C.P. 07320; dtolentinolsro@gmail.com

(2) Investigadora Titular, Ingeniería Estructural, Instituto de Ingeniería, UNAM, Coyoacán, 04510 Ciudad de México. Teléfono: (55) 562336 54; sruiz@iingen.unam.mx 


\section{INTRODUCCIÓN}

La seguridad de un sistema estructural se provee especificando capacidades a los elementos estructurales que implícitamente cumplen con un nivel de confiabilidad aceptable; sin embargo, el sistema estructural continuamente está expuesto a condiciones de carga de eventos naturales (ej. sismo, viento, oleaje, inundación, etc.). Al término de estos eventos, el sistema estructural sufre cierto deterioro en sus propiedades mecánicas dando lugar a que se debilite su capacidad estructural y al mismo tiempo se incremente su demanda estructural ante cierto nivel de solicitación. Como resultado de lo anterior, el valor de la confiabilidad del sistema estructural se reduce a medida que pasa el tiempo, llegando hasta el punto en el que dicho sistema presente niveles de confiabilidad no deseados. Lo anterior hace ver la necesidad de desarrollar criterios que permitan evaluar la confiabilidad estructural en el tiempo con el fin de conocer qué tan segura está la estructura a lo largo de su vida útil.

Dada la importancia de evaluar el estado de las estructuras que sufren deterioro, varios autores han propuesto diversos criterios para evaluar dicho estado en término de niveles de confiabilidad, por ejemplo: a) mediante procesos de Markov (Montes et al., 2003; Straub, 2009); b) simulación de Monte Carlo (Díaz y Esteva, 1997); c) simulación direccional (Melchers, 1992; Mori y Ellinwood, 1993) y d) aproximaciones basadas en métodos FORM/SORM (Rackwitz, 2001). Por otro lado, Frangopol et al., (2004) hacen una revisión general sobre modelos probabilistas para evaluar el ciclo de vida de estructuras que presentan deterioro estructural; sin embargo, ninguno de estos enfoques evalúa la confiabilidad en términos del factor de confianza al término de un intervalo de tiempo.

El factor de confianza, $\lambda_{\text {conf }}$, propuesto por Cornell et al. (2002), se introdujo originalmente para evaluar la confiabilidad estructural de edificios de acero. Posteriormente, la formulación se extiende para tomar en cuenta: a) la variación en el tiempo de la capacidad estructural (Torres y Ruiz, 2007), y b) la variación en el tiempo tanto de la capacidad como la demanda estructural, dada una intensidad (Tolentino et al., 2012); sin embargo, las formulaciones anteriores consideran que la capacidad estructural varía en el tiempo de manera lineal.

En este trabajo se propone un criterio para evaluar la confiabilidad en términos del factor de

confianza, $\lambda_{\text {conf }}$, y su correspondiente nivel de confianza, $K_{x}$, por medio del desarrollo de expresiones matemáticas cerradas. En la formulación se considera que la degradación de la capacidad estructural se puede expresar mediante una función no-lineal en el intervalo total de tiempo. El criterio se ilustra en un edificio de concreto reforzado de 10 niveles sujeto a cargas sísmicas.

\section{FACTOR DE CONFIANZA SIN CONSIDERAR EL DETERIORO ESTRUCTURAL}

Una manera de calcular la tasa media anual de falla es separando las incertidumbres que involucran a la demanda sísmica de la capacidad estructural como sigue (Cornell, 1968; Esteva, 1968; Cornell et al., 2002):

$$
\begin{aligned}
& v_{D}(d)=\int-\frac{d v(y)}{d y}(P(D \geq d y) d y \\
& E\left(v_{F}\right)=\int-\frac{d v_{D}(d)}{d d}(P(C \leq d) d d
\end{aligned}
$$


donde $v_{D}(d)$ representa la curva de peligro de demanda estructural; $d v(y) / d y$ representa la derivada de la curva de peligro sísmico, $P(D \geq d \mid y)$ es la probabilidad condicional de que la demanda estructural, $D$, se exceda un cierto valor $d$, dada una intensidad sísmica $y ; E\left(v_{F}\right)$ representa la tasa anual de falla estructural; $d v_{D}(d) / d d$ es la derivada de la curva de peligro de demanda, y $P(C \leq d)$ es la probabilidad de que la capacidad estructural $C$ sea menor que o igual a un valor dado, $d$.

Las ecuaciones 1 y 2 pueden ser resueltas por medio de integración numérica o por medio de métodos simplificados. Con la finalidad de resolver las expresiones antes mencionadas se han propuesto las siguientes consideraciones utilizando un enfoque simplificado como sigue (Kennedy y Short, 1994; Cornell et al., 2002):

a) La curva de peligro, $v(y)$, se representa para la intensidad de interés mediante la función $v(y)=k y^{-r}$, donde $k$ y $r$ son parámetros que definen la forma de la curva de peligro sísmico.

b) La mediana de la demanda estructural, $\hat{D}$, para la región de intensidad de interés, se puede representar como función de la intensidad, $y$, mediante la expresión $\hat{D}=a y b$. Esta se considera que presenta una distribución de tipo lognormal con desviación estándar del logaritmo natural igual a $\sigma_{\ln D \mid y}($ Rosenblueth y Esteva, 1972).

c) La capacidad estructural del estado límite de interés presenta una mediana de la capacidad, $\hat{C}$, de tipo lognormal, con desviación estándar del logaritmo natural igual a $\sigma_{\ln C}$.

A partir de las consideraciones $a, b$ y $c$, la ecuación 1 se transforma como sigue (Kennedy y Short, 1994):

$v_{D}(d)=v\left(\frac{d}{a}\right)^{\frac{1}{b}} \exp \left(\frac{r^{2}}{2 b^{2}}\left(\sigma_{\ln D \mid y}^{2}\right)\right)$

Considerando las simplificaciones $a$ y $c$, la tasa media anual de falla, $E\left(v_{F}\right)$, (ecuación 2) se expresa como (Jalayer y Cornell, 2003):

$E\left(v_{F}\right)=k\left(y_{c}\right)^{-r} \exp \left(\frac{r^{2}}{2 b^{2}}\left(\sigma_{\ln D \mid y}^{2}+\sigma_{\ln C}^{2}\right)\right)$

Introduciendo las incertidumbres epistémicas, el valor esperado de la tasa media anual de falla obtenida por Cornell et al. (2002) es igual a:

$E\left(v_{F}\right)=k\left(y_{\hat{C}}\right)^{-r} \exp \left\{\frac{r^{2}}{2 b^{2}}\left[\sigma_{\ln D \mid y}^{2}+\sigma_{\ln C}^{2}+\sigma_{U D}^{2}+\sigma_{U C}^{2}\right]\right\}$

donde $y_{C}=\left[\frac{\hat{C}}{a}\right]^{\frac{1}{b}}$ es la intensidad de la solicitación asociada con la mediana del estado límite de la capacidad, $\hat{C} ; \sigma^{2}{ }_{\ln D \mid y}$ y $\sigma_{\ln C}^{2}$ son las varianzas de los logaritmos naturales de la demanda estructural, $D$, 
y de la capacidad estructural, $C$, correspondientes al estado límite de interés; $\sigma_{D U}^{2}$ y $\sigma_{C U}^{2}$ representan las varianzas de las incertidumbres epistémicas asociadas a la demanda y a la capacidad, respectivamente; $a$ y $b$ son parámetros que definen la forma de la mediana de la demanda estructural. Con la finalidad de contar con una formulación que resulte familiar para los ingenieros, Cornell et al. (2002) establecen que la tasa media anual de falla, $v_{F}$, sea igual a un valor permisible, $v_{0}$. De acuerdo a lo anterior y haciendo algunos arreglos algebraicos, se obtiene la siguiente expresión:

$\phi \hat{C} \geq \gamma \hat{D}^{v_{0}}$

donde $\phi=\exp \left[-\frac{r}{2 b}\left(\sigma_{\ln C}^{2}+\sigma_{C U}^{2}\right)\right] ; \gamma=\exp \left[\frac{r}{2 b}\left(\sigma_{\ln D y}^{2}+\sigma_{D U}^{2}\right)\right] ; \hat{D}^{v_{0}}$ representa la mediana de la demanda para una intensidad dada $y_{v_{0}}$, que a su vez se define como el nivel de intensidad, $y$, con probabilidad anual, $v_{0}$, de ser excedida, y se expresa como sigue:

$\hat{D}^{v_{0}}=a\left(y_{v_{0}}\right)^{b}$

A partir de la formulación anterior, se deduce el factor de confianza, $\lambda_{\text {conf }}$. Dicho factor indica el nivel de confiabilidad que presenta un sistema estructural ante las solicitaciones externas. Cuando $\lambda_{\text {conf }}$ excede la unidad indica que hay una mayor confianza de que la estructura tenga un comportamiento deseado. Si $\lambda_{\text {conf }}$ es menor que la unidad indica que dicha confianza no es la deseable. El grado de confianza para que se presente dicho factor de confianza se expresa mediante el nivel de confianza, $K_{x}$.

$\lambda_{\text {conf }}=\frac{\phi \hat{C}}{\gamma \hat{D}^{v_{0}}}$

El nivel de confianza para que se presente el factor de confianza se expresa como sigue:

$$
K_{x}=\frac{\ln \left\lfloor\lambda_{c o n f}\right\rfloor}{\sigma_{U T}}+\frac{r \sigma_{U T}}{2 b}
$$

donde $K_{x}$ es una variable Gaussiana estandarizada con probabilidad $x$ de no ser excedida, por lo que $x$ representa el grado de confianza, y $\sigma_{U T}=\sqrt{\left(r^{2} / b^{2}\right)\left(\sigma_{U D}^{2}+\sigma_{U C}^{2}\right)}$. La ecuación anterior implica que el número anual de fallas, $v_{F}$, asociadas a un nivel de confianza $x$, está dado por (Cornell et al., 2002):

$v_{F \mid x}=\hat{v}_{F} \exp \left(K_{x} \sigma_{U T}\right)=k\left(y_{\hat{C}}\right)^{-r} \exp \left(\frac{r^{2}}{2 b^{2}}\left(\sigma_{\ln D \mid y}^{2}+\sigma_{\ln C}^{2}\right)\right) \exp \left(K_{x} \sigma_{U T}\right)$

donde $\hat{v}_{F}$ representa la mediana de la tasa media anual de falla, $v_{F}$. Las ecuaciones 8, 9 y 10 se utilizan en los lineamientos del SAC/FEMA para la evaluación de estructuras. 


\section{FACTOR DE CONFIANZA EN FUNCIÓN DEL TIEMPO}

En lo que sigue se extiende la metodología presentada en la sección anterior para el caso en el que las propiedades mecánicas de los elementos estructurales cambian en el tiempo, y como consecuencia decrece el valor de la capacidad estructural y aumenta la demanda estructural ante una secuencia de solicitaciones que se presente en cierto intervalo de tiempo.

La tasa media anual de falla, $E\left(v_{F}\right)$, es igual al número esperado de fallas, $\eta_{F}$, cuando $\Delta t \rightarrow 1$. Se hace notar que el número esperado de fallas es una extensión de la tasa media anual de falla. En lo que sigue se utilizará el concepto del número esperado de fallas al término de un intervalo de tiempo $[t, t+\Delta t)$. De acuerdo a lo anterior, el número esperado de fallas asociado a un nivel de confianza $x$, en el intervalo de tiempo $[t, t+\Delta t)($ similar a la ecuación 10$)$, se expresa como sigue:

$\eta_{F \mid x}(t, \Delta t)=\eta_{F}(t, \Delta t) \exp \left(K_{x \mid t} \sigma_{U T \mid t}\right) \Omega(t, \Delta t)$

donde:

$\eta_{F}(t, \Delta t)=k\left(y_{\hat{C} \mid t}\right)^{-r} \exp \left[\frac{r^{2}}{2 b^{2}}\left(\sigma^{2}{ }_{\ln D \mid y, t}+\sigma^{2}{ }_{\ln C \mid t}\right)\right]$

donde $\eta_{F}(t, \Delta t)$ es la mediana del número esperado de fallas para el intervalo de tiempo $[t, t+\Delta t) ; \Omega(t, \Delta t)$ es el factor de corrección del número esperado de fallas al término del intervalo de tiempo $[t, t+\Delta t) ; \mathrm{y}$ $\eta_{F \mid x}(t, \Delta t)$ representa el número de fallas correspondiente a un nivel de confianza $x$, dentro del intervalo $[t, t+\Delta t)$.

En el formato Demand and Capacity Factor Design (DCFD, Cornell et al., 2002) se asume que la tasa anual de falla, $v_{F}$, debe ser igual que una tasa anual permisible, $v_{0}$. De manera similar, en este estudio se establece que el número esperado de fallas asociado a un grado de confianza $x$, al término de un intervalo del tiempo $[t, t+\Delta t)$ debe ser igual a cierto valor de tasa media anual permisible $v_{0}$ en el tiempo, por lo que la ecuación 11 se expresa como:

$\eta_{F \mid x}(t, \Delta t)=\eta_{F}(t, \Delta t) \exp \left(K_{x \mid t} \sigma_{U T \mid t}\right) \Omega(t, \Delta t)=v_{0} \Delta t$

Resolviendo y separando términos con respecto a la ecuación anterior, se llega a la siguiente expresión del factor de confianza, $\lambda_{\text {conf }}$, y su correspondiente nivel de confianza, $K_{x}$, al final del intervalo de tiempo $[t, t+\Delta t)$ como:

$$
\begin{aligned}
& \lambda_{\text {conf }}(t, \Delta t)=\frac{\phi \hat{C}}{\gamma \hat{D}^{v_{0}}}\left[\frac{\Omega(t, \Delta t)}{\Delta t}\right]^{-\frac{b}{r}} \\
& K_{x}(t, \Delta t)=\frac{\ln \left\lfloor\lambda_{c o n f}(t, \Delta t)\right\rfloor}{\sigma_{U T}}+\frac{r \sigma_{U T}}{2 b}
\end{aligned}
$$




\section{CASO EN EL QUE SE CONSIDERA QUE LA CAPACIDAD (NO-LINEAL) Y LA DEMANDA ESTRUCTURAL VARÍAN SIMULTÁNEAMENTE EN EL TIEMPO}

La confiabilidad estructural expresada en términos del número esperado de fallas al final del intervalo de tiempo $[t, t+\Delta t)$ puede expresarse como una extensión de la ecuación 5, como sigue:

$\eta_{F}(t, \Delta t)=\int_{t}^{t+\Delta t} k\left(y_{C, \tau}\right)^{-r} \exp \left\{\frac{r^{2}}{2 b(\tau)^{2}}\left[\sigma_{\ln D \mid y}^{2}(\tau)+\sigma_{\ln C}^{2}(\tau)+\sigma_{U D}^{2}(\tau)+\sigma_{U C}^{2}(\tau)\right]\right\} d \tau$

donde $y_{\hat{C}, \tau}=\left[\frac{\hat{C}(\tau)}{a(\tau)}\right]^{-\frac{1}{b(\tau)}}$ es la intensidad de la solicitación asociada con la mediana de la capacidad en el tiempo $\tau ; \sigma_{\ln D y}^{2}(\tau)$ y $\sigma_{\ln C}^{2}(\tau)$ son las varianzas del logaritmo natural de la demanda y de la capacidad, en el tiempo $\tau ; \sigma_{U D}^{2}(\tau)$ y $\sigma_{U C}^{2}(\tau)$ representan las varianzas de las incertidumbres epistémicas relacionadas con la demanda estructural $(D)$ y con la capacidad $(C)$ en el tiempo.

Con la finalidad de considerar de manera simultánea la capacidad estructural (no-lineal) y la demanda estructural, se hacen las siguientes hipótesis:

d) La varianza del logaritmo natural tanto de la capacidad, $\sigma_{\ln C}^{2}(\tau)$, como de la demanda, $\sigma_{\ln C}^{2}(\tau)$, se consideran constantes en el tiempo, es decir, $\sigma_{\ln C}^{2}(\tau)=\sigma_{\ln C}^{2}$ y $\sigma_{\ln D \mid y}^{2}(\tau)=\sigma_{\ln D \mid y}^{2}$, respectivamente.

e) Los parámetros $a(\tau)$ y $b(\tau)$ son constantes en el intervalo de tiempo de interés, por lo que $a(\tau)=a$ y $b(\tau)=b$, respectivamente.

f) La mediana de la capacidad estructural varía en el tiempo como una función polinomial de segundo grado, dada por:

$\hat{C}(\tau)=\alpha+\beta \tau+\gamma \tau^{2}$

g) La mediana de la demanda estructural, $\hat{D}$, varía en el tiempo $\tau$, como sigue:

$\hat{D}(\tau)=(a+f \tau) y^{b}+g \tau$

Tomando en cuenta las consideraciones $a, c, d, e, f$ y $g$ e integrando la ecuación 16, se obtiene la siguiente expresión cerrada para estimar el número esperado de fallas al término del intervalo de tiempo $[t, t+\Delta t)$ :

$$
\eta_{F P}(t, \Delta t)=k\left(\frac{\alpha+\beta^{\prime} t+\gamma^{2}}{a+f t}\right)^{-\frac{r}{b}} \exp \left(\frac{r^{2}}{2 b^{2}}\left(\sigma_{\ln D \mid y}^{2}+\sigma_{\ln C}^{2}+\sigma_{U D}^{2}+\sigma_{U C}^{2}\right)\right) \Omega_{P}(t, \Delta t)
$$


donde:

$$
\begin{aligned}
& \left.\left.\Omega_{P}(t, \Delta t)=\frac{b(a+f t)}{f(b-r)}\left(1-\frac{2(a+f t) \gamma}{2 a \gamma+f\left(-\beta^{\prime}+\sqrt{\beta^{\prime 2}-4 \alpha \gamma}\right.}\right)\right)^{\frac{r}{b}}\left(1+\frac{2(a+f t) \gamma}{-2 a \gamma+f\left(\beta^{\prime}+\sqrt{\beta^{\prime 2}-4 \alpha \gamma}\right.}\right)\right)^{\frac{r}{b}} \cdots \\
& {\left[-F_{1}\left(X ; Y, Y^{\prime} ; Z ; u(t), v(t)\right)+F_{1}\left(X ; Y, Y^{\prime} ; Z ; u(t+\Delta t), v(t+\Delta t)\right)\left(1+\frac{f \Delta t}{a+f t}\right)\left(\frac{\alpha+\beta^{\prime} t+t^{2}}{a+f t}\right)^{\frac{r}{b}} \cdots\right.} \\
& \left.\left(1-\frac{2 f \Delta t \gamma}{f\left(-\beta^{\prime}+\sqrt{\beta^{\prime 2}-4 \alpha \gamma}\right)-2 f t \gamma}\right)^{\frac{r}{b}}\left(\frac{\alpha+\beta^{\prime}(t+\Delta t)+\gamma(t+\Delta t)^{2}}{a+f(t+\Delta t)}\right)^{-\frac{r}{b}}\left(1+\frac{2 f \Delta t \gamma}{f\left(\beta^{\prime}+\sqrt{\beta^{\prime 2}-4 \alpha \gamma}\right)+2 f t \gamma}\right)^{\frac{r}{b}}\right]
\end{aligned}
$$

donde $\Omega_{P}(t, \Delta t)$ representa el factor de corrección del número esperado de fallas que considera la variación en el intervalo $[t, t+\Delta t)$ de la demanda sísmica y la capacidad estructural como una función polinomial; $\beta^{\prime}=\beta-g$. La función de Appell, $F_{1}\left(X ; Y, Y^{\prime} ; Z ; u, v\right)$, implícita en la ecuación 20 se resuelve como sigue (Slater, 1966):

$$
F_{1}\left(X ; Y, Y^{\prime} ; Z ; u, v\right)=\sum_{p=0}^{\infty} \sum_{q=0}^{\infty} x^{p} y^{q} \frac{\frac{\Gamma(p+q+X)}{\Gamma(X)} \frac{\Gamma(p+Y)}{\Gamma(Y)} \frac{\Gamma\left(q+Y^{\prime}\right)}{\Gamma\left(Y^{\prime}\right)}}{\frac{\Gamma(p+q+Z)}{\Gamma(Z)} p ! q !}
$$

donde:

$$
\begin{aligned}
& \left.X=\frac{b+r}{b} ; Y=\frac{r}{b}, Y^{\prime}=\frac{r}{b} ; Z=2+\frac{r}{b} ; u(t)=\frac{2 a \gamma}{2 a \gamma-f\left(\beta^{\prime}+\sqrt{\beta^{\prime 2}-4 \alpha \gamma}\right)}, v(t)=\frac{2 a \gamma}{2 a \gamma+f\left(-\beta^{\prime}+\sqrt{\beta^{\prime 2}-4 \alpha \gamma}\right.}\right) \\
& u(t+\Delta t)=\frac{2 \gamma(a+f \Delta t)}{2 a \gamma-f\left(\beta^{\prime}+\sqrt{\beta^{\prime 2}-4 \alpha \gamma}\right)}, v(t+\Delta t)=\frac{2 \gamma(a+f \Delta t)}{2 a \gamma+f\left(-\beta^{\prime}+\sqrt{\beta^{\prime 2}-4 \alpha \gamma}\right)} ; \Gamma(.) \text { es la función gamma; }
\end{aligned}
$$

$Y, Y^{\prime}, u$ y $v$ son números reales; $X$ y $Z$ deben ser números enteros.

Siguiendo las mismas suposiciones para llegar a la ecuación 13, se tiene:

$$
k\left(\frac{\alpha+\beta^{\prime} t+\gamma t^{2}}{a+f t}\right)^{-\frac{r}{b}} \exp \left(\frac{r^{2}}{2 b^{2}}\left(\sigma_{\ln D \mid y}^{2}+\sigma_{\ln C}^{2}+\sigma_{U D}^{2}+\sigma_{U C}^{2}\right)\right) \exp \left(K_{x \mid t} \sigma_{U T}\right) \Omega_{P}(0, \Delta t)=v_{0} \cdot \Delta t
$$

De la ecuación 22 es posible obtener el factor de confianza que considera la variación de la capacidad no-lineal (función de segundo orden) y la demanda estructural de manera simultánea, para el intervalo de tiempo de interés, como sigue:

$$
\lambda_{\text {confP }}(t, \Delta t)=\frac{\phi \hat{C}}{\gamma \hat{D}^{\nu_{0}}}\left[\frac{\Omega_{P}(t, \Delta t)}{\Delta t}\right]^{-\frac{b}{r}}
$$




\section{CASO EN EL QUE SE CONSIDERA QUE LA CAPACIDAD (LINEAL) Y LA DEMANDA ESTRUCTURAL VARÍAN SIMULTÁNEAMENTE EN EL TIEMPO}

Con la finalidad de conocer la variación tanto de la capacidad estructural (lineal) como de la demanda sísmica, dada una intensidad, en el intervalo de tiempo $[t, t+\Delta t)$, Tolentino et al., (2012) consideran lo siguiente:

h) La mediana de la capacidad, $\hat{C}$, varía linealmente durante el intervalo de tiempo de interés, como sigue:

$$
\hat{C}(\tau)=\alpha+\beta \tau, \beta<0
$$

Considerando las suposiciones $a, c, d, e, f$ y $h$ e integrando la ecuación 16, el número esperado fallas que considera la variación de manera simultánea la capacidad (lineal) y de la demanda estructural al final del intervalo de interés, es igual a:

$$
\eta_{F, L}(t, \Delta t)=k\left(\frac{\alpha+\beta^{\prime} t}{a+f t}\right)^{-\frac{r}{b}} \exp \left(\frac{r^{2}}{2 b^{2}}\left(\sigma_{\ln D \mid y}^{2}+\sigma_{\ln C}^{2}+\sigma_{U D}^{2}+\sigma_{U C}^{2}\right)\right) \Omega_{L}(t, \Delta t)
$$

donde:

$$
\begin{aligned}
& \Omega_{L}(t, \Delta t)=\frac{b\left(\alpha+\beta^{\prime} t\right)}{(b-r) \beta^{\prime}}\left(\frac{(a+f t) \beta^{\prime}}{-f \alpha+a \beta^{\prime}}\right)^{-\frac{r}{b}}\left[-F(A, B ; C ; x(t))+F(A, B ; C ; x(t+\Delta t))\left(\frac{\alpha+\beta^{\prime} t}{a+f t}\right)^{\frac{r}{b}} \cdots\right. \\
& \left.\left(1+\frac{\beta^{\prime} \Delta t}{\alpha+\beta^{\prime} t}\right)\left(\frac{\alpha+\beta^{\prime}(t+\Delta t)}{a+f(t+\Delta t)}\right)^{-\frac{r}{b}}\left(1+\left(\frac{\beta^{\prime} f \Delta t}{(a+f t) \beta^{\prime}}\right)^{-\frac{r}{b}}\right)\right]
\end{aligned}
$$

donde $\Omega_{L}(t, \Delta t)$ es un factor de corrección del número esperado de fallas que considera la variación tanto de la capacidad estructural (lineal) como de la demanda sísmica, dada una intensidad $[t, t+\Delta t)$. La función hipergeométrica, $F(A, B ; C ; x)$, implícita en la ecuación 26 se resuelve mediante la siguiente serie hipergeométrica (Ford, 1955; Agnew, 1960; Rainville, 1961):

$$
F(A, B ; C ; x)=1+\frac{A B}{1 ! C} x+\frac{A(A+1) B(B+1)}{2 ! C(C+1)} x^{2}+\cdots+\frac{A(A+1) \cdots(A+n-1) B(B+1) \cdots(B+n-1)}{C(C+1) \cdots(C+n-1) n !} X^{n}
$$

donde $A=1-\frac{r}{b} ; B=-\frac{r}{b} ; C=2-\frac{r}{b} ; x(t)=\frac{f\left(\beta^{\prime} t+\alpha\right)}{f \alpha-a \beta^{\prime}} ; x(t+\Delta t)=\frac{f\left[\beta^{\prime}(t+\Delta t)+\alpha\right]}{f \alpha-a \beta^{\prime}}$. Los parámetros $A$, $C$ y $x$ pueden tomar cualquier número real, $B$ debe ser un número real entero.

Siguiendo un procedimiento similar para llegar a la ecuación 23, el factor de confianza que considera la variación tanto de la capacidad estructural como de la demanda sísmica, al término del intervalo de tiempo $[t, t+\Delta t)$, se expresa como: 


$$
\lambda_{\text {confL }}(t, \Delta t)=\frac{\phi \hat{C}}{\gamma \hat{D}^{v_{0}}}\left[\frac{\Omega_{L}(t, \Delta t)}{\Delta t}\right]^{-\frac{b}{r}}
$$

donde $\lambda_{\text {confL }}(t, \Delta t)$ es el factor de confianza al final del intervalo de tiempo que considera la variación de capacidad estructural (lineal) y la demanda sísmica al término del intervalo de tiempo $[t, t+\Delta t)$.

\section{CASO EN EL QUE SE CONSIDERA QUE LA CAPACIDAD (LINEAL) VARÍA EN EL TIEMPO, MIENTRAS QUE LA DEMANDA ESTRUCTURAL ES INDEPENDIENTE DEL TIEMPO}

Con la finalidad de considerar la variación en el tiempo de la capacidad estructural y suponiendo que la demanda estructural es constante, Torres y Ruiz (2007) resolvieron la ecuación 16 considerando las suposiciones $a, b, c, d, e, \mathrm{y} h$. El resultado es el siguiente:

$\eta_{F C}(t, \Delta t)=k\left(\frac{\alpha+\beta t}{a}\right)^{-\frac{r}{b}} \exp \left(\frac{r^{2}}{2 b^{2}}\left(\sigma_{\ln D \mid y}^{2}+\sigma_{\ln C}^{2}+\sigma_{U D}^{2}+\sigma_{U C}^{2}\right)\right) \Omega_{C}(t, \Delta t)$

donde:

$\Omega_{C}(t, \Delta t)=\frac{b(\alpha+\beta t)}{(b-r) \beta}\left[-1+\left(1+\frac{\beta \Delta t}{\alpha}\right)^{1-\frac{r}{b}}\right]$

donde $\Omega_{C}(t, \Delta t)$ es el factor de corrección del número esperado de fallas que considera el deterioro de la capacidad estructural mientras que la demanda permanece constante en el intervalo de tiempo de interés. Suponiendo la misma consideración que se siguió para obtener la ecuación $23, \lambda_{\text {confc }}(t, \Delta t)$ queda como sigue:

$\lambda_{\text {confC }}(t, \Delta t)=\frac{\phi \hat{C}}{\gamma \hat{D}^{v_{0}}}\left[\frac{\Omega_{C}(t, \Delta t)}{\Delta t}\right]^{-\frac{b}{r}}$

Con la ecuación anterior se evalúa el factor de confianza al término del intervalo de interés cuando sólo se considera el deterioro estructural de la capacidad, suponiendo que la demanda estructural no varía en el tiempo.

\section{EJEMPLO}

Se obtiene el factor de confianza, $\lambda_{\text {conf }}(t, \Delta t)$, y su respectivo nivel de confianza, $K_{x}(t, \Delta t)$, correspondiente a un edificio de concreto reforzado ubicado en la zona IIIb del Valle de México. El edificio es una estructura regular a base de marcos de concreto reforzado, este cuenta con 10 niveles y 3 crujías de $5 \mathrm{~m}$ en ambos sentidos (ver figura 1). La estructura se diseñó de acuerdo con los requerimientos que establece las Normas Técnicas Complementarias de 1976 para una ductilidad $Q=4$, se utilizó un coeficiente sísmico de diseño $C_{s}=0.24$. Se consideró una resistencia a la compresión del concreto $f_{c}^{\prime}=200 \mathrm{~kg} / \mathrm{cm}^{2}$ y un esfuerzo de fluencia del acero $f_{y}=4200 \mathrm{~kg} / \mathrm{cm}^{2}$. En la figura 2 se muestran las secciones nominales y su 
respectivo refuerzo. Para efectuar el análisis no lineal se consideró un marco exterior que tiene un periodo fundamental $T=1.03 \mathrm{~s}$.
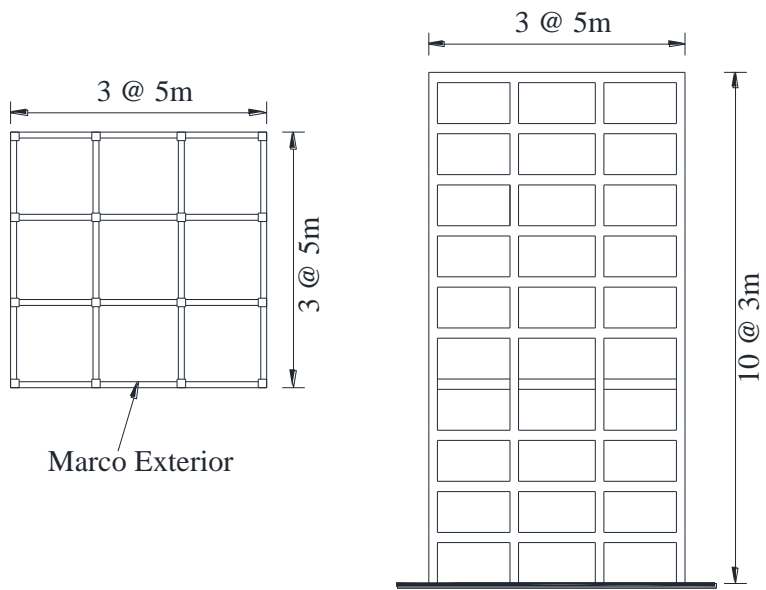

Figura 1. Planta y elevación del marco en estudio.
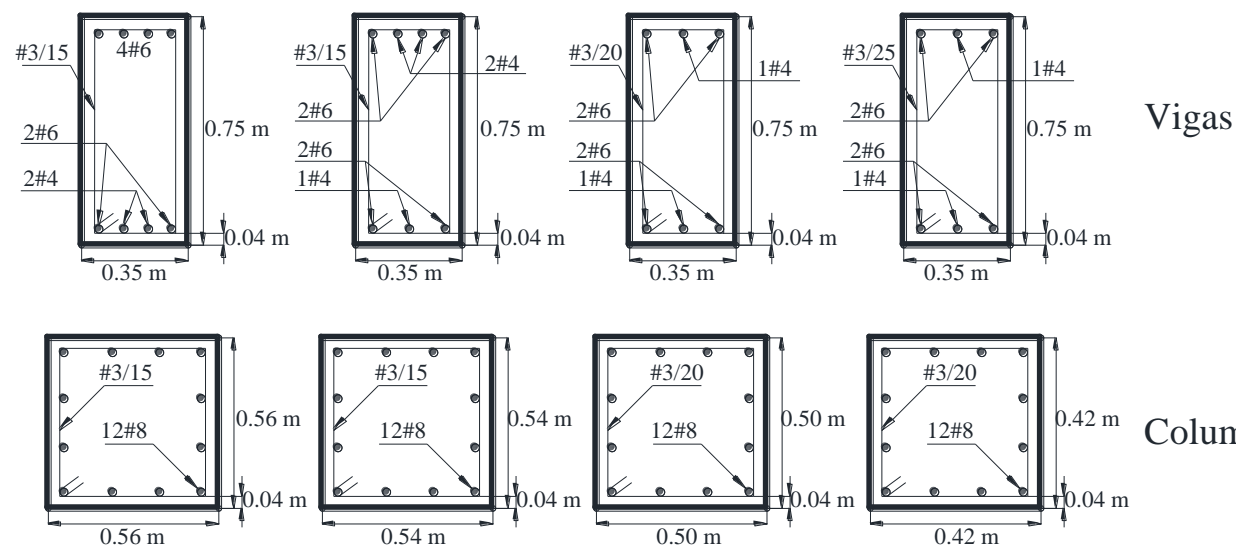

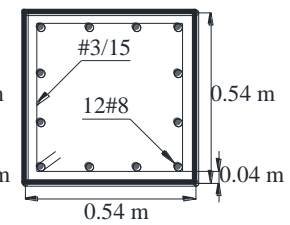
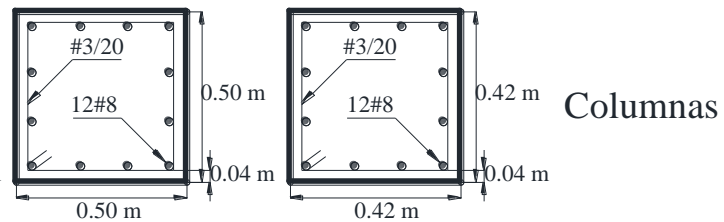

Entrepiso 1-4

Entrepiso 5-6

Entrepiso 7-8

Entrepiso 9-10

Figura 2. Secciones y acero de refuerzo de los elementos que integran el marco estructural.

\section{SIMULACIÓN DE ACELEROGRAMAS}

La carga extraordinaria que históricamente ha producido daños considerables y casos de colapso en la zona geográfica en la que se ubica el sistema estructural en estudio, son los movimientos sísmicos. Dada la escasa información de registros sísmicos en el sitio en estudio es necesario generar movimientos sísmicos sintéticos. Para la simulación de los acelerogramas sintéticos se consideró que el movimiento sísmico que se simula sigue un proceso estocástico ergódico Gaussiano no-estacionario. Se simularon 100 acelerogramas sintéticos a partir de los registro obtenidos en la estación de la Secretaría de Comunicaciones y Transportes (SCT) el 19 de septiembre de 1985 (Grigoriu et al.,1988).

\section{SIMULACIÓN DE INTENSIDADES Y TIEMPOS DE ESPERA}

Con la finalidad de evaluar la evolución del daño estructural ante secuencias sísmicas es necesario simular tanto la ocurrencia de eventos sísmicos como su intensidad. Con base en lo anterior, se simularon 
intensidades a partir de la curva de peligro sísmico asociado al periodo fundamental de la estructura. La curva de peligro sísmico corresponde al sitio de SCT con un porcentaje de amortiguamiento crítico igual a $5 \%$.

La ocurrencia de los eventos sísmicos se supuso como un proceso estocástico de Poisson, por lo que sus tiempos de espera presentan una distribución de probabilidades de tipo exponencial. Para la simulación de los tiempos de espera se supuso que el número medio de tiempos de espera entre eventos sísmicos intensos es igual a 5 años.

Se construyeron 20 historias de eventos simulados a partir de la simulación de intensidades y tiempos de espera considerando un intervalo de tiempo de 150 años. En este estudio sólo se seleccionaron intensidades mayores a $100 \mathrm{~cm} / \mathrm{seg}^{2}$ ya que se observó que intensidades menores producían daño despreciable. Con base en lo anterior, se presentaron 8,11 y 15 intensidades mayores a $100 \mathrm{~cm} / \mathrm{seg}^{2}$ al término de 50, 100, y 150 años. En la figura 3 se muestra un ejemplo de historia de eventos simulados, se hace notar que se presentan 4 intensidades mayores a $100 \mathrm{~cm} / \mathrm{seg}^{2}$, mismas que representan 0,2 y 4 secuencias sísmicas al termino de 50, 100 y 150 años, respectivamente.

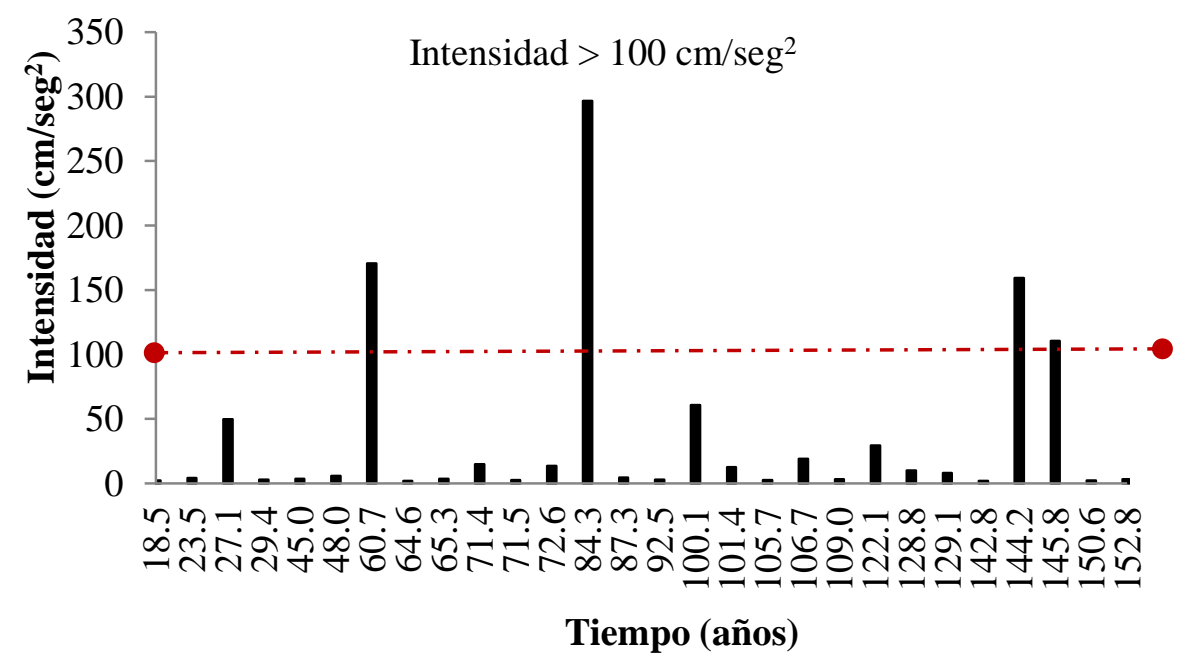

Figura 3. Ejemplo de historia de eventos simulados (tiempos de espera e intensidades).

\section{CAPACIDAD ESTRUCTURAL EN EL TIEMPO}

Con la finalidad de evaluar la evolución de la capacidad estructural a causa del daño acumulado por secuencias sísmicas, al término de un intervalo de tiempo, se sometió a la estructura a una serie de análisis dinámicos no lineales "paso a paso". Con base en las 20 historias de eventos simulados (descritas en la sección anterior), se asoció aleatoriamente un aceleragrama sintético a cada intensidad mayor a $100 \mathrm{~cm} / \mathrm{seg}^{2}$. El acelerograma seleccionado se escaló de manera que la intensidad simulada correspondiera a la intensidad que presenta su espectro de pseudoaceleracion para $\mathrm{T}=1.03 \mathrm{seg}$ (periodo fundamental de la estructura). Posterior a las secuencias sísmicas, se aplicó una aceleración monotónicamente creciente simulando la acción de un análisis estático no-lineal. En los casos de historias de eventos simulados en donde no se presentaron intensidades mayores a $100 \mathrm{~cm} / \mathrm{seg}^{2}$, sólo se aplicó una aceleración monotónicamente creciente. Los análisis dinámicos no lineales se llevaron a cabo en el programa Drain 2D (Campos y Esteva, 1997) donde se incorpora un modelo histérico que considera la degradación de rigidez y resistencia. Se consideró que la falla en la estructura se presenta en el instante cuando se articulan todas las vigas y columnas de un 
entrepiso. En el caso del edificio en estudio, el tercer entrepiso resultó ser el crítico. En las figuras 4 a, b y c se muestran las curvas de capacidad (CC) con daño acumulado para los intervalos de 50, 100 y 150 años después de construida la estructura.

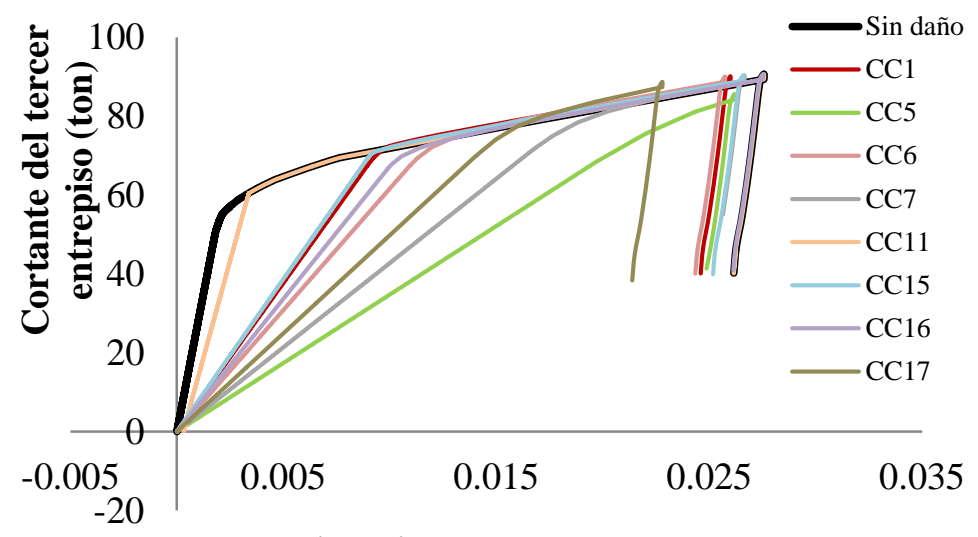

Distorsión máxima del tercer entrepiso

a) 50 años

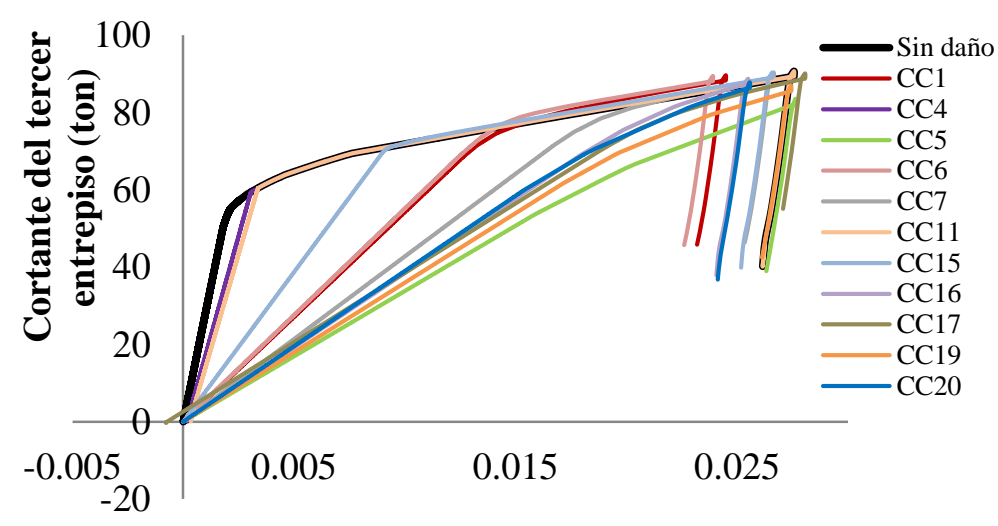

Distorsión máxima del tercer entrepiso

b) 100 años

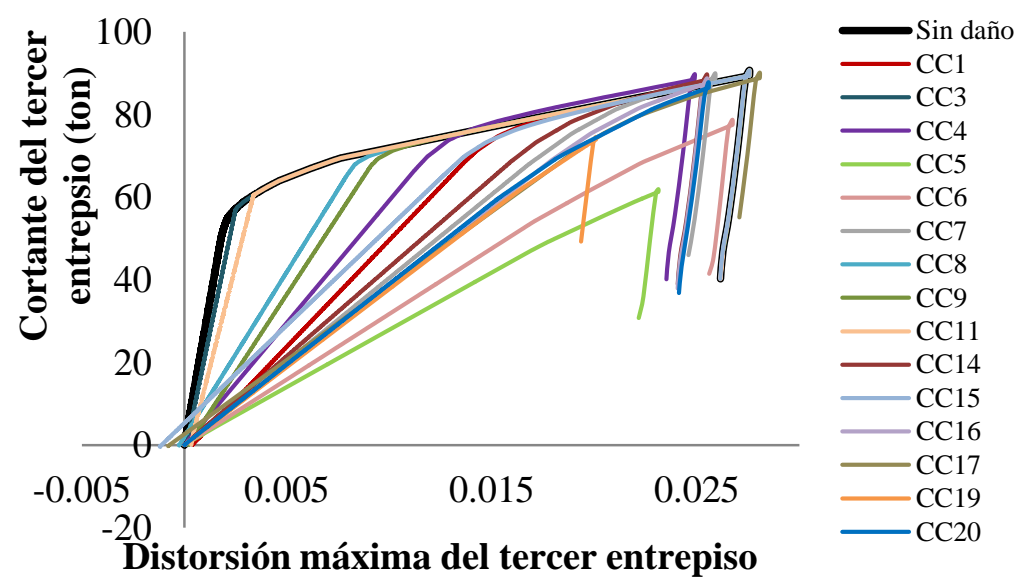

c) 150 años

Figura 4. Curvas de capacidad al término de 50, 100 y 150 años después de construida la estructura. 
En la figura 4 se hace notar la disminución de rigidez y resistencia en las curvas de capacidad a medida que se incrementa el intervalo de tiempo de análisis y con esto, una mayor probabilidad de que se presente un intensidad mayor o igual al umbral de intensidad $\left(100 \mathrm{~cm} / \mathrm{seg}^{2}\right)$ que produce daño a la estructura. La figura 4a muestra 8 curvas de capacidad, $C C$, dado que sólo ocurren 8 intensidades mayores a 100 $\mathrm{cm} / \mathrm{seg}^{2}$, para el caso de 100 y 150 años (figura 4 b y c) se presentan 11 y 15 curvas de capacidad.

La figura 5 muestra los valores de la mediana de la capacidad al término de los intervalos de tiempo en estudio (0, 50, 100 y 150 años) considerando que dichos valores siguen una distribución de tipo lognormal. En este estudio se caracteriza la degradación de la capacidad estructural en el tiempo como un polinomio de segundo grado (no-lineal) y alternativamente, como una función lineal. En la figura 5 se muestran los valores de ajuste de ambos criterios no-lineal (ecuación 17) y lineal (ecuación 24) a lo largo del intervalo de tiempo en estudio. Los valores de la desviación estándar del logaritmo natural de la capacidad, $\sigma_{\ln C}$, se calcularon a partir de la figura 4, resultando valores de 0, 0.046, 0.064 y 0.099 para los intervalos de tiempo de 0, 50, 100 y 150 años.

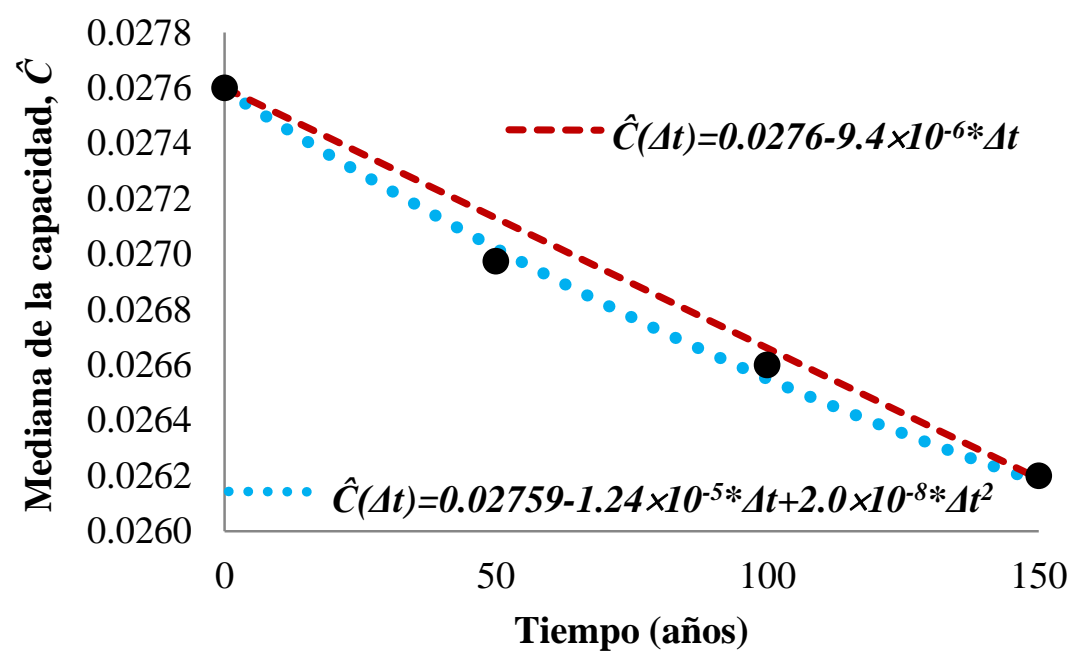

Figura 5. Mediana de la capacidad, $\hat{C}$, al término de diferentes intervalos de tiempo.

\section{DEMANDA ESTRUCTURAL SÍSMICA EN EL TIEMPO}

La demanda sísmica al término de distintos intervalos de tiempo se obtuvo mediante análisis dinámicos no lineales "paso a paso". Para su obtención, se utilizaron las mismas historias de eventos simulados que se emplearon para obtener la capacidad estructural en el tiempo, sólo que al final de cada intervalo en estudio, se cambió la carga monotónicamente creciente por un acelerograma sintético elegido aleatoriamente, y este se fue escalando hasta que la estructura presentó la falla incipiente. En la figura 6 se muestran los valores de la mediana de la demanda sísmica al término de 0, 50, 100 y 150 años. Con base en los resultados, se verificó que dichos valores obedecieran a una distribución de tipo lognormal. La figura 6 muestra que se presentan valores de daño inicial acumulado al término de 50, 100 y 150 años y este se incrementa a medida que aumenta el intervalo de tiempo. Dicho daño se debe al efecto de las secuencias sísmicas. 


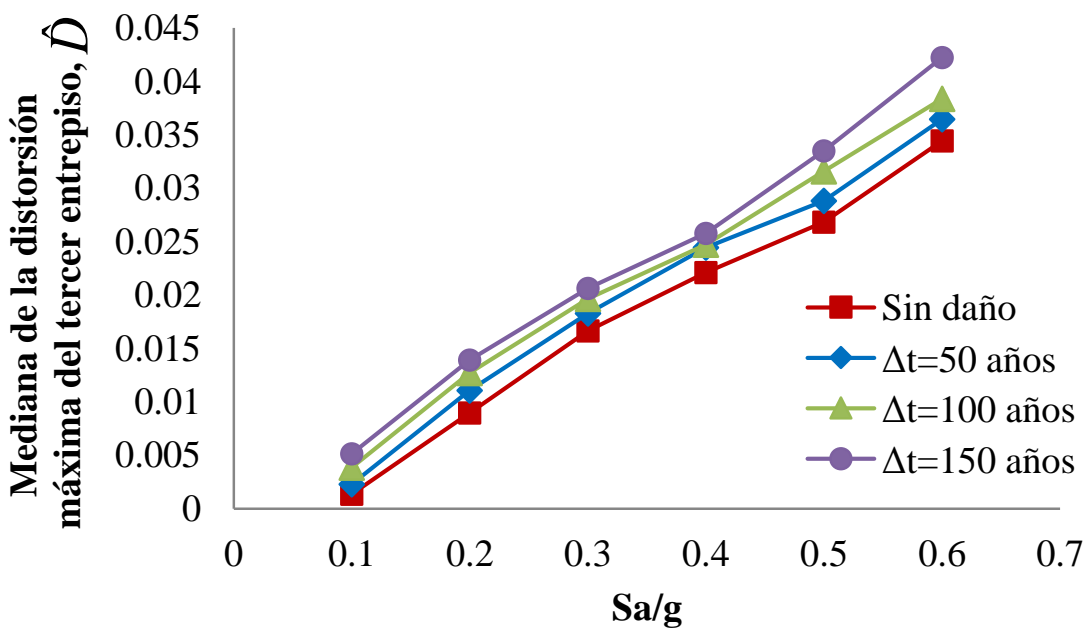

Figura 6. Mediana de la demanda sísmica al término de diferentes intervalos de tiempo.

Las figuras $7 \mathrm{a}, \mathrm{b}, \mathrm{c}$ y d muestran los ajustes de los valores de la mediana de la demanda al término de $0,50,100$ y 150 años, respectivamente. Se utilizó la ecuación 18 para llevar a cabo el ajuste de dichos valores. Con base en la ecuación 18, los parámetros $a, f$ y $b$ definen la forma de los valores de la mediana de la demanda, dada una intensidad, mientras que el parámetro $g$ define el valor del daño inicial acumulado. Dada la similitud en tendencia con los valores de la mediana de la demanda, los valores de las desviaciones estándar del logaritmo natural de la demanda, $\sigma_{\operatorname{In} D \mid y}$, se ajustaron con la ecuación 18, resultando la siguiente expresión: $\sigma_{\ln D \mid y}=\left(0.3+1.5 \times 10^{-4} \cdot \Delta t\right) \cdot y^{0.52}+2.9 \times 10^{-4} \cdot \Delta t$. Sustituyendo el intervalo de tiempo de interés en la expresión anterior y considerando la intensidad que produce la falla incipiente, resulta lo siguiente $\sigma_{\ln D \mid 0}=0.24, \sigma_{\ln D \mid 50}=0.25, \sigma_{\ln D \mid 100}=0.27 \mathrm{y} \sigma_{\ln D \mid 150}=0.29$.

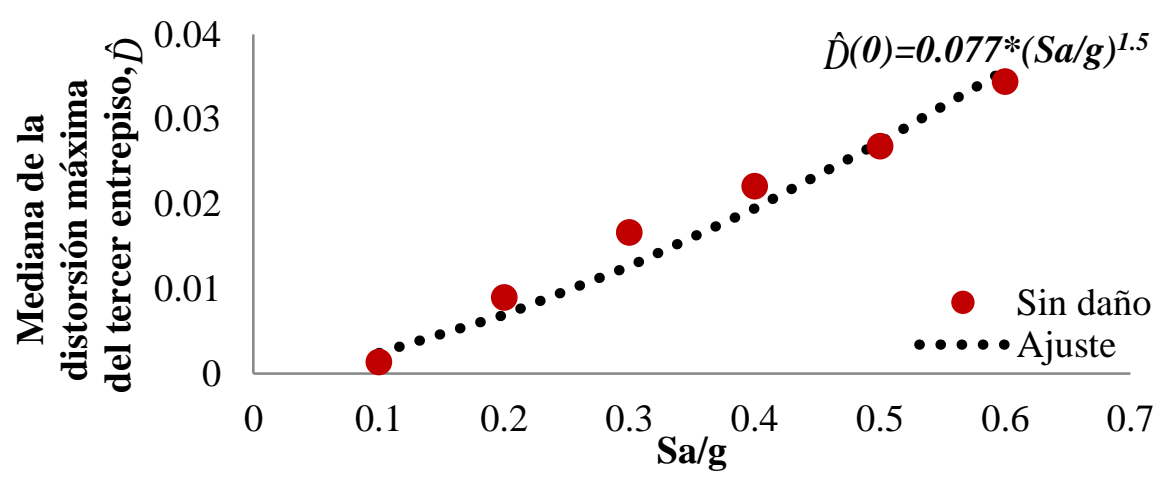

a) 0 años

Figura 7. Ajuste de los valores de la mediana de la demanda sísmica en el tiempo. 


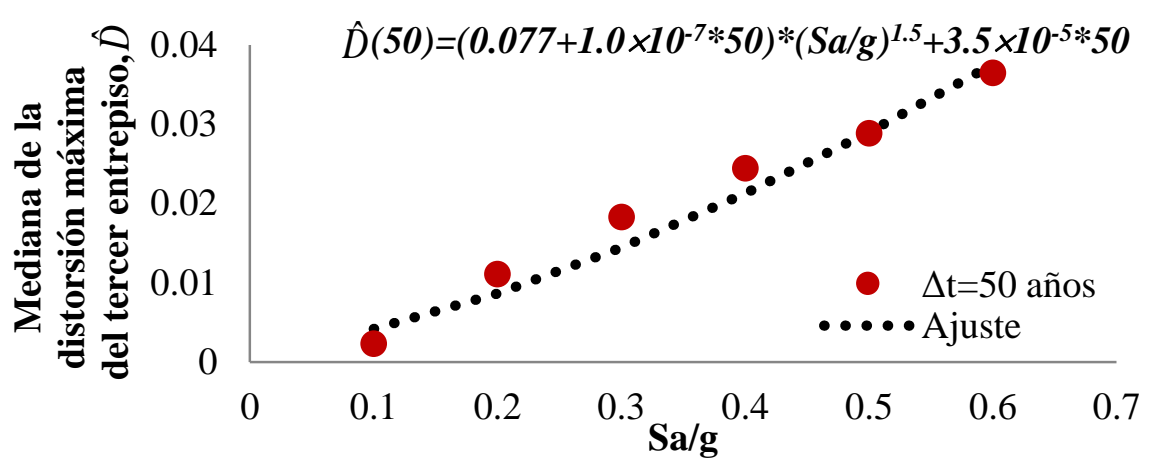

b) 50 años

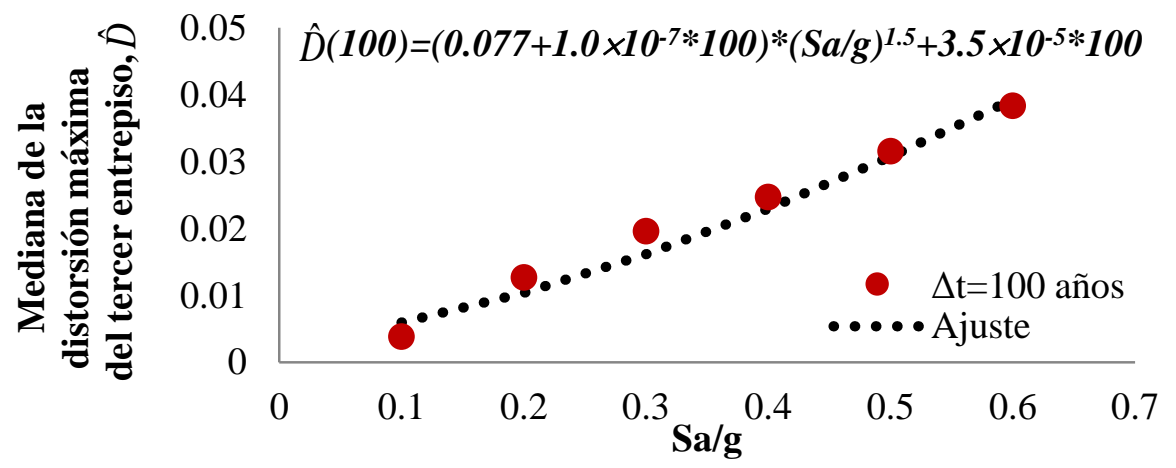

c) 100 años

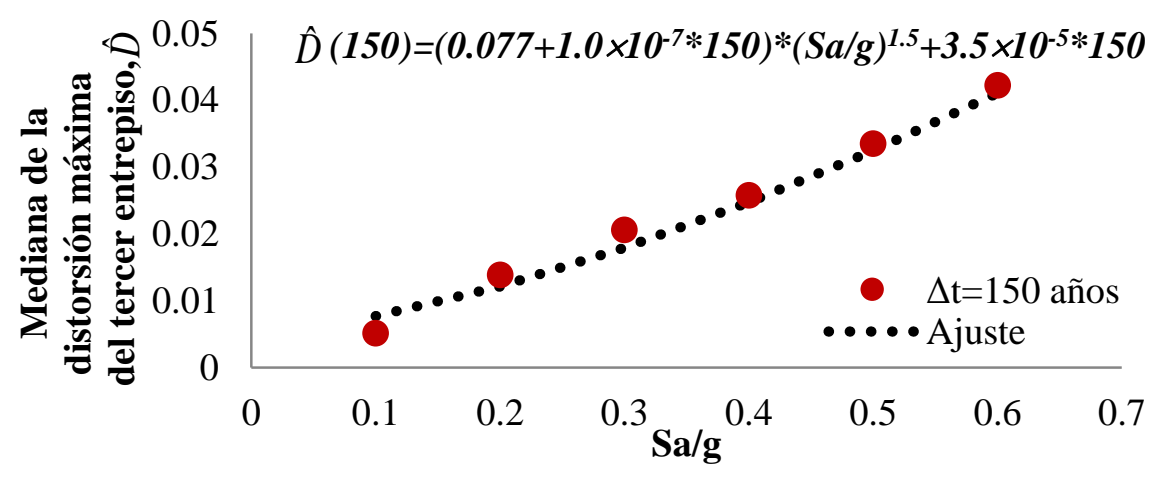

d) 150 años

Figura 7 (Continuación). Ajuste de los valores de la mediana de la demanda sísmica en el tiempo.

\section{FACTOR DE CORRECCIÓN EN EL TIEMPO}

Para evaluar el factor de corrección en el tiempo es necesario conocer el valor de los parámetros de las ecuaciones 20, 26 y 30. Los parámetros $\alpha, \beta$ y $\gamma$ se presentan en la figura 5, los valores que ajustan la forma de la mediana de la demanda $a, f$ y $b$ se muestran en la figura 7 , y los parámetros que ajustan la forma de la curva de peligro sísmico para la intensidad de interés, tienen un valor igual a $k=1.7 \times 10^{-4}$ y $r=3.75$. A juicio de los autores, se consideró un valor de 0.2 en las varianzas epistémicas, $\sigma_{D U}^{2}$, y aleatorias, $\sigma_{C U}^{2}$. La figura 8 muestra el factor de corrección correspondiente a los tres casos de estudio. 


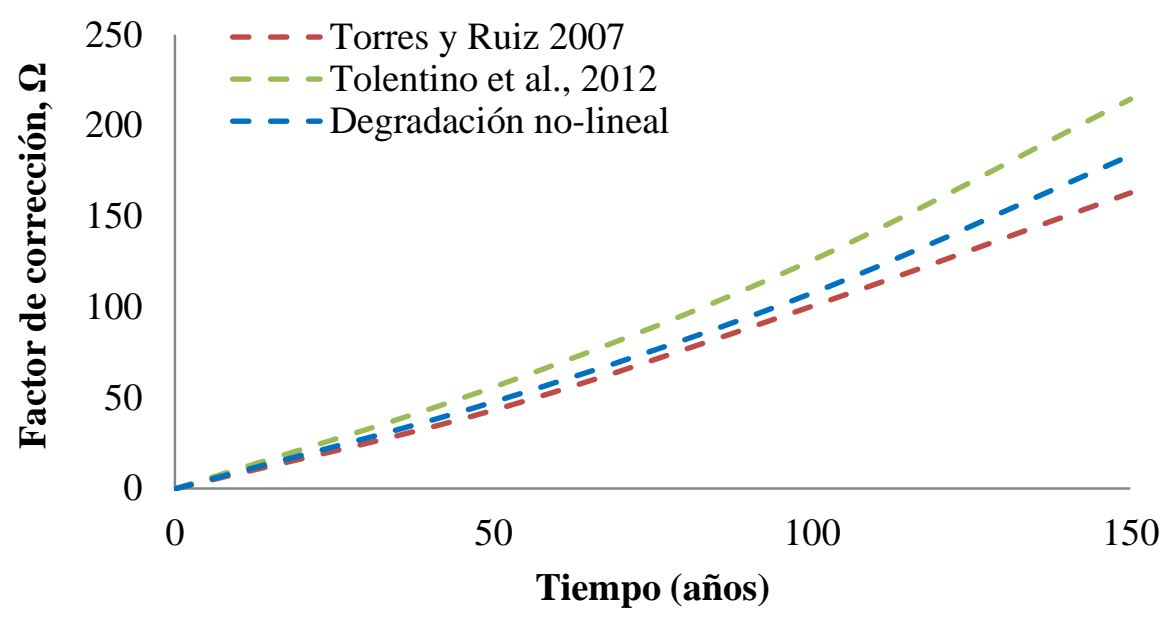

Figura 8. Factor de corrección correspondiente a los diferentes casos de estudio.

La figura 8 muestra diferencias del $22.6 \%, 20.1 \%, 24 \%$ y del $9.4 \%, 6.7 \%, 11.3 \%$ al término de 50, 100 y 150 años cuando no se considera la variación de la demanda sísmica en el tiempo (Torres y Ruiz, 2007) comparado con los criterios que sí consideran dicha variación (Tolentino et al., 2012) y este estudio. Las diferencias entre los criterios que consideran tanto la variación de la capacidad como la demanda, al término de un intervalo de tiempo (ecuaciones 22 y 28), resultaron del orden de 17.1, 16.7 y 18.2 al término de 50,100 y 150 años.

\section{EVALUACIÓN DE LA CONFIABILIDAD ESTRUCTURAL EN EL TIEMPO}

El factor de confianza, $\lambda_{\text {conf }}(t, \Delta t)$, (ver figura 9) al término de distintos intervalos de tiempo, se obtiene para los siguientes casos: a) no se considera el daño acumulado, b) considerando la variación de la capacidad (lineal) mientras que la demanda sísmica es independiente del tiempo, c) considerando la variación tanto de la capacidad estructural (lineal) y la demanda en el tiempo y d) considerando que varía en el tiempo tanto la capacidad (no-lineal) como la demanda. La figura 9 muestra diferencias de 4.2, 9.5 y $15.8 \%$ del factor de confianza obtenido con el criterio de Torres y Ruiz (2007) con respecto al criterio que no considera daño acumulado. Las diferencias entre el criterio que no considera daño con respecto al criterio que considera la variación tanto de la demanda como de la capacidad (lineal), y alternativamente con el criterio que considera la variación de la demanda y la capacidad (no-lineal) en el tiempo resultan del orden de $14.8 \%, 33.9 \%, 59.3 \%$ y del $10.8 \%, 26.5 \%, 49.7 \%$ al término de 50, 100 y 150 años.

En la figura 10 se muestran el nivel de confianza al término de diferentes instantes de tiempo considerando los casos: a) sin daño (Cornell et al., 2002), b) Sólo varía la capacidad estructural (lineal) en el tiempo (Torres y Ruiz, 2007), c) varía tanto la capacidad (lineal) como la demanda en el tiempo, y d) varía la capacidad (no-lineal) y la demanda en el tiempo. La figura 10 muestra un disminución del grado de confianza del $87.7 \%, 83.7 \%$ y 78.5\% para el criterio que sólo considera la variación de la capacidad en el tiempo, dicha pequeña disminución es como se esperaba dado que no se considera la incertidumbre que introduce el considerar la variación de la demanda sísmica en el tiempo. Con respecto a los casos que sí consideran la demanda sísmica, se tiene un disminución del $79.3 \%, 60.8 \%$ y $36.7 \%$ para el caso que considera la variación (lineal) de la capacidad y la demanda en el tiempo, y del $84.8 \%, 68.3 \%$ y $45.2 \%$ para el caso desarrollado en este estudio. 


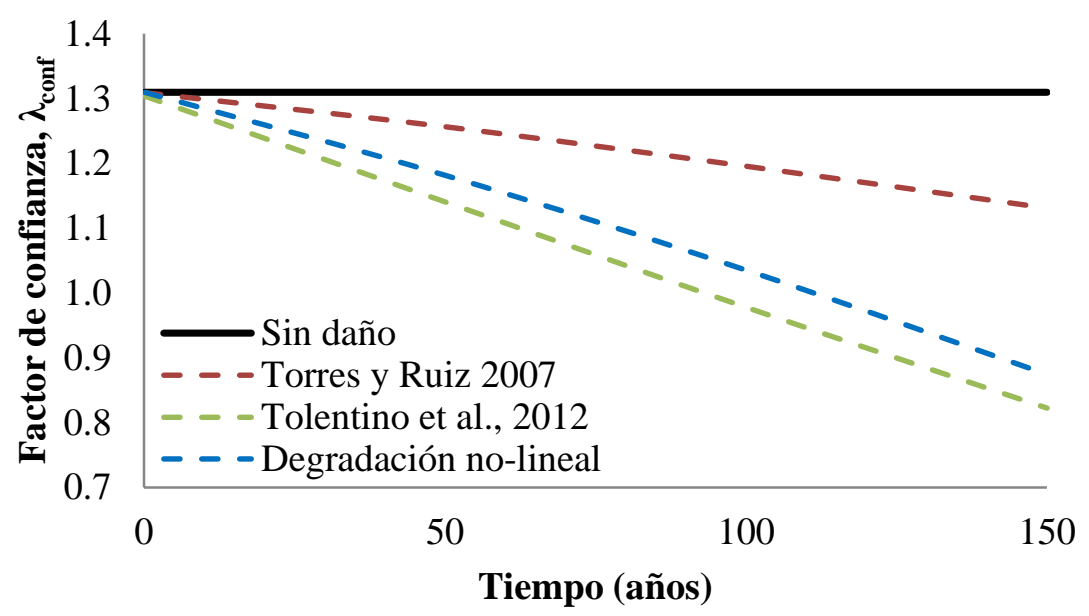

Figura 9. Factor de confianza al término de distintos intervalos de tiempo.

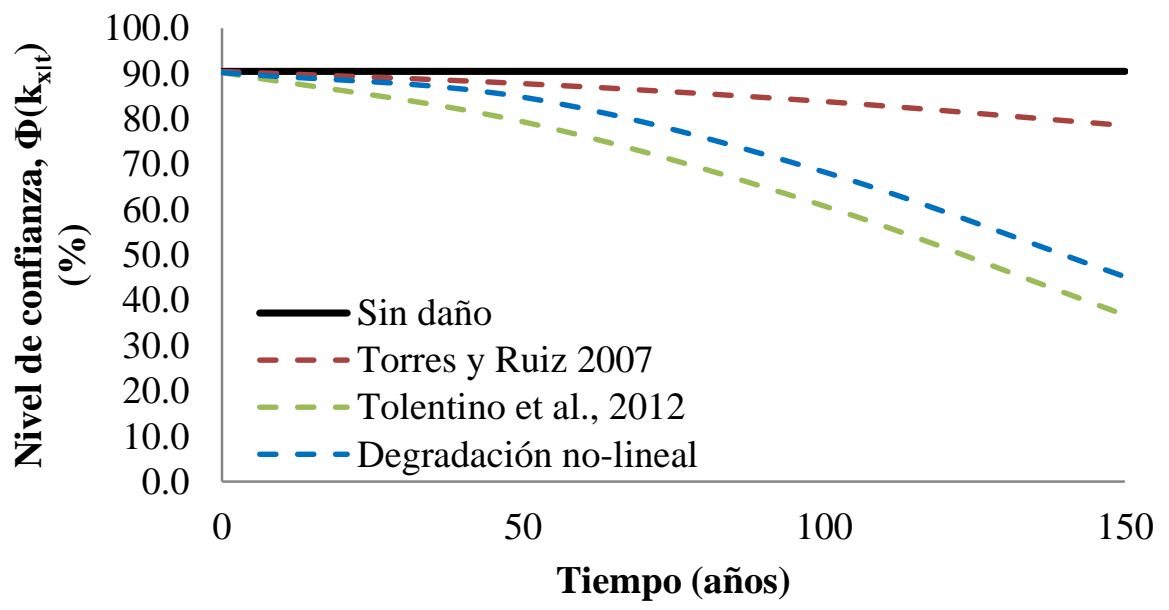

Figura 10. Nivel de confianza al término de distintos intervalos de tiempo.

\section{CONCLUSIONES}

Se propone un criterio para evaluar el factor de confianza y su correspondiente nivel de confianza que considera la variación de la demanda sísmica y la capacidad (mediante una función tipo polinomio de segundo grado), al término de un intervalo de tiempo. La formulación que se propone utiliza expresiones matemáticas cerradas, con formato similar a las expresiones descritas en los códigos de diseño. Dicho factor se evalúa para un edificio de concreto reforzado sujeto a secuencias sísmicas para los siguientes casos: a) no se considera el daño en el tiempo, b) sólo se considera que varía la capacidad (lineal) en el tiempo, c) se considera que la capacidad (lineal) y la demanda varían simultáneamente en el tiempo, y d) varían tanto la capacidad (no-lineal) como la demanda en el tiempo.

Se observó que el modelo de degradación no-lineal de la capacidad estructural se apega al comportamiento del edificio comparado con el modelo de degradación lineal de la capacidad estructural. Las diferencias de porcentajes entre estos criterios son del orden de $3.5 \%, 5.5 \%$ y $6 \%$ al término de 50 , 100 y 150 años. Se puede decir que estos porcentajes representan una diferencia pequeña en términos de 
ingeniería estructural; sin embargo, para que se presente el mismo valor de factor de confianza en ambos criterios, tuvieron que transcurrir 20 años; por ejemplo, $\lambda_{\text {conf }}=1$ se presenta en el año 91 y en el año 110 después de construida la estructura cuando se considera el criterio propuesto. En este sentido, la diferencia en tiempo es significativa y esto repercutiría notablemente en el establecimiento de políticas de diseño y/o mantenimiento estructural. Con base en lo anterior, se recomienda evaluar en factor de confianza con el criterio que se propone en el presente estudio.

Las expresiones propuestas pueden aplicarse a cualquier tipo de sistema estructural (ej. edificios, torres de transmisión, puentes, plataforma marinas, plantas nucleares, tanques de almacenamiento, etc.), sometido a una o múltiples cargas ambientales (ej. sismo, viento, oleaje, etc.), y pueden considerar diferentes estados límite. El criterio propuesto se puede extender para establecer criterios de diseño para un nivel confiabilidad pre-escrito (ej. normas de diseño para plataformas marinas fijas), por otro lado, se pueden establecer planes de mantenimiento para la búsqueda de intervalos de tiempo óptimo de inspección y mantenimiento.

\section{AGRADECIMIENTOS}

Se agradece a CONACYT, a la Universidad Nacional Autónoma de México y al Instituto Politécnico Nacional por el apoyo recibido.

\section{REFERENCIAS}

Agnew, R P (1960), Differential equations, McGraw -Hill Book Company Japan, pp. 268-270.

Campos, D y L Esteva (1997), "Modelo de comportamiento histerético y de daño para vigas de concreto reforzado", XI Congreso Nacional de Ingeniería Sísmica, Veracruz, México.

Cornell, C A (1968), "Engineering seismic hazard analysis", Bulletin of the Seismological Society of America, Vol. 58, No.5, pp. 1583-1606.

Cornell, C A, F Jalayer, R O Hamburger y D A Foutch (2002), "The probabilistic basis for the 2000 SAC/FEMA steel moment frame guidelines", Journal of Structural Engineering, Vol. 128, No. 4, pp. 526-533.

Díaz, O J y L Esteva (1997), "Confiabilidad de estructuras y daño acumulado", Séptimas Jornadas Chilenas de Sismología e Ingeniería Antisísmica, La Serena, Chile, pp. 589-598.

Esteva, L (1968), "Bases para la formulación de decisiones de diseño sísmico", Tesis de Doctorado, Facultad de Ingeniería, UNAM, México.

Ford, L R (1955), Differential Equations, McGraw -Hill Book Company, New York, USA.

Frangopol, D M, M J Kallen y J M Van Noortwijk, (2004), "Probabilistic models for life-cycle performance of deteriorating structures: review and future directions", Progress in Structural Engineering and Materials, Vol. 6, pp. 197-212.

Grigoriu, M, S E Ruiz y E Rosenblueth (1988), "Nonstationary models of seismic ground acceleration", Earthquake Spectra, Vol. 4, pp. 551-568. 
Jalayer, F y C A Cornell (2003), “A technical framework for probability-based demand and capacity factor design (DCFD) seismic formats", Pacific Earthquake Engineering Research Center, PEER Report 2003/08, University of California, Berkeley.

Kennedy, R C y S A Short (1994), "Basis for seismic provisions of DOE-STD-1020", Report UCRL-CR111478, BNL-52418, Lawrence Livermore Nat. Lab., University of California, Livermore, CA. and Brookhaven Nat. Lab., Upton, NY.

Melchers, R E (1992), "Load-space formulation for time-dependent structural reliability", Journal of Engineering Mechanics, Vol. 118, No. 5, pp. 853-870.

Montes, R, E Heredia y L Esteva (2003), "Optimal maintenance strategies for structures in seismic zones", Earthquake Engineering and Structural Dynamics, Vol. 32, pp. 245-264.

Mori, Y y B R Ellingwood (1993), "Time-dependent system reliability analysis by adaptive importance sampling”, Structural Safety, Vol. 12, No. 1, pp. 59-73.

Rackwitz, R (2001), “Reliability analysis: a review and some perspectives”, Structural Safety, Vol. 23, pp. 365-395.

Rainville, E D y P E Bedient (1981), Elementary differential equations, London: Collier Macmillan Publishers, pp. 392-395.

Rosenblueth, E y L Esteva (1972), "Reliability basis for some Mexican codes", Probabilistic Design of Reinforced Concrete Buildings, ACI Publication, SP-31, pp. 1-41.

Slater, L J (1966), Generalized hypergeometric functions, London: Cambridge University Press, pp. 210215.

Straub, D (2009), "Stochastic modeling of deterioration processes through dynamic Bayesian networks", Journal of Engineering Mechanics, Vol. 135, No.10, pp. 1089-1099.

Tolentino, D, S E Ruiz y M A Torres (2012), "Simplified closed-form expressions for the mean failure rate of structures considering structural deterioration", Structure and Infrastructure Engineering, Vol. 8, No. 5, pp. 483-496.

Torres, M A y S E Ruiz (2007), "Structural reliability evaluation considering capacity degradation over time”, Engineering Structures, Vol. 29, pp. 2183-2192. 